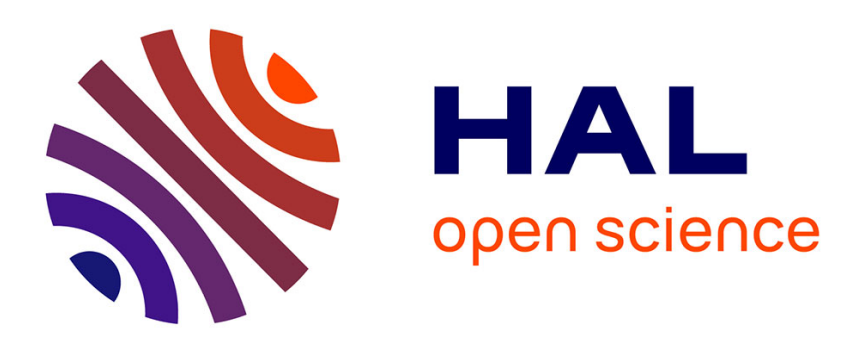

\title{
Linearized harmonic balance based derivation of slow flow for some class of autonomous single degree of freedom oscillators
}

Serge Bruno Yamgoué, T.C. Timoléon Crépin Kofané

\section{- To cite this version:}

Serge Bruno Yamgoué, T.C. Timoléon Crépin Kofané. Linearized harmonic balance based derivation of slow flow for some class of autonomous single degree of freedom oscillators. International Journal of Non-Linear Mechanics, 2008, 43 (9), pp.993. 10.1016/j.ijnonlinmec.2008.05.001 • hal-00501788

\author{
HAL Id: hal-00501788 \\ https://hal.science/hal-00501788
}

Submitted on 12 Jul 2010

HAL is a multi-disciplinary open access archive for the deposit and dissemination of scientific research documents, whether they are published or not. The documents may come from teaching and research institutions in France or abroad, or from public or private research centers.
L'archive ouverte pluridisciplinaire HAL, est destinée au dépôt et à la diffusion de documents scientifiques de niveau recherche, publiés ou non, émanant des établissements d'enseignement et de recherche français ou étrangers, des laboratoires publics ou privés. 


\section{Author's Accepted Manuscript}

Linearized harmonic balance based derivation of slow flow for some class of autonomous single degree of freedom oscillators

Serge Bruno Yamgoué, Timoléon Crépin Kofané

PII: S0020-7462(08)00094-2

DOI: doi:10.1016/j.ijnonlinmec.2008.05.001

Reference: $\quad$ NLM 1480

Reference:

To appear in: International Journal of Non-

\section{Linear Mechanics}

Received date: 10 October 2007

Revised date: 18 April 2008

Accepted date: 2 May 2008

Cite this article as: Serge Bruno Yamgoué and Timoléon Crépin Kofané, Linearized harmonic balance based derivation of slow flow for some class of autonomous single degree of freedom oscillators, International Journal of Non-Linear Mechanics, doi:10.1016/j.ijnonlinmec.2008.05.001

This is a PDF file of an unedited manuscript that has been accepted for publication. As a service to our customers we are providing this early version of the manuscript. The manuscript will undergo copyediting, typesetting, and review of the resulting galley proof before it is published in its final citable form. Please note that during the production process errors may be discovered which could affect the content, and all legal disclaimers that apply to the journal pertain. 


\title{
Linearized harmonic balance based derivation of slow flow for some class of autonomous single degree of freedom oscillators
}

\author{
Serge Bruno Yamgoué and Timoléon Crépin Kofané \\ Laboratoire de Mécanique, Département de Physique, Faculté de Sciences, \\ Université de Yaoundé I, B.P. 812 Yaoundé Cameroun
}

\begin{abstract}
In this paper we exploit the embedding of linearization in the harmonic balance method developed by $\mathrm{Wu}$ and its collaborators to propose an approach for deriving the slow flow for some class of damped autonomous single degree of freedom oscillators. The linearized harmonic balance method is used to compute the coefficients of the harmonics of an assumed form of the solution and to derive a system of two coupled ordinary differential equations related to the slow flow. A power series procedure is next used to decouple the coupled system and to obtain the slow flow. Two examples provided to illustrate the proposed procedure show excellent results.
\end{abstract}




\section{Introduction}

The development of methods applicable to the determination of approximate analytical solutions to oscillator equations of the form

$$
\ddot{x}+f(x, \dot{x})=0, \quad x(0)=\rho_{0}, \quad \dot{x}(0)=0
$$

has witnessed a renewed interest in recent years. Here, single and double overdots denote respectively first and second derivatives with respect to time $t, x=x(t) \in \mathbb{R}$, and $f$ is a nonlinear function of its arguments. See Refs. [1, 2, 4, 3, 5, 6, 7, 8, 9, 10, 11, 12, 13, 14, 15] for a sample of the related works. We can identify three valuable motivations that sustain this interest. Indeed, it is well known on the one hand that standard approximate techniques such as the Lindstedt-Poincaré (LP) method, the Krylov-Bogoliubov-Mitropolsky (KBM) and the method of multiple scales (MMS) [16] have two principal limitations. The first is that they apply only to problems for which there is a linear part and the second is that the nonlinear part must be small with respect to the linear one. Overcoming these limitations appears as a first motivation to the current interest in the development of analytical techniques. This is the case of Ref. [4] and Refs. $[5,7]$ which propose a combination of the LP method with, respectively, the technique of expansion of constant and the linear $\delta$-expansion. On the other hand, there exists some techniques that are much more general and thus 
do not suffer the above limitations; but whose application usually involves quite intractable mathematics. The current attention devoted to methods of analytical approximation is also motivated by the aim of simplifying the complexity of the mathematics resulting from those methods. A remarkable example here is the embedding of the Newton procedure in the harmonic balance method $[2,3,6,8,9,10]$. The last motivation to the actual interest is simply to propose novel methods $[1,11,13,15]$. Some of these are specially designed and work very efficiently for systems with uncommon restoring force, such as those with fractional powers [13].

We remark that almost all of the modified or new methods so published up to now are built exclusively in the spirit of strictly periodic or limit cycle oscillations. Thus, although they generally perform considerably better than the classical methods for that type of problems, they are still far, in their actual form, from completely superseding the MMS or the KBM method. In fact, for the important class of damped nonlinear oscillators, the novel methods cannot even compete with them due to their lack of means of handling modulation. This ability may appear at first glance to be of little interest if one considers only single degree of freedom oscillators, Eq.(1). In this case, the modulations are due only to damping; and the asymptotic motions are known to be either fixed points or limit cycles. Furthermore, the modulation equations or slow flow that can be derived 
are usually not integrable.

The derivation of the slow flow is however of great importance for the analysis of stability in the case of limit cycles. Moreover, the analysis of resonant responses of oscillators to external periodic excitation is often started from the approximate solutions of their autonomous motions. In this case, one may capture more of the dynamics with the solutions of damped oscillations than with the solutions of periodic oscillations [17]. More importantly, the ability of handling modulation is indispensable for the expected application of the new methods to (even undamped) coupled oscillators. One could then take advantage of their more accurate approximations of large amplitude oscillations in the important problem of traveling waves in one dimensional lattices. The adaptation of the new techniques to the study of modulation in damped single degree of freedom oscillators is undoubtedly an important and necessary step toward the complete problem of high-amplitude waves modulation in one dimensional lattice.

This paper builds upon the linearized harmonic balance method $[2,3,6$, $8,9,10]$ to suggest an approach for deriving the slow flow for some subclass of Eq.(1). The systems considered here are characterized by nonlinear functions $f$ such that

$$
f(-x,-\dot{x})=-f(x, \dot{x})
$$


and there exists a positive integer $k$ such that

$$
\frac{\partial^{j} f}{\partial x^{j}}(0,0)=0, \quad 0 \leq j<k ; \quad \frac{\partial^{k} f}{\partial x^{k}}(0,0)>0 .
$$

Mickens [18] pointed out that Eq.(2a) should hold for the harmonic balance (and its linearized version to be used below) to provide consistent approximations to the symmetric periodic and/or limit cycle solutions of Eq.(1). By condition (2b), we further restrict our discussion to systems for which the origin is a stable equilibrium when dissipation is neglected. Thus, systems described by equations such as, for example, $\ddot{x}+\delta \dot{x}+\sigma^{2}\left(x^{3}-x\right)=0$ are excluded although for $\delta=0$ the linearized harmonic balance applies to their large amplitude periodic solutions. The reason for this exclusion is that, being concerned with damped motions, condition (2a) will no more be satisfied for these oscillations when they settle in one of the asymmetric well of the potential under the effect of dissipation.

The basic idea is presented in the following section and is exemplified using viscously damped oscillators in Sec. 3. Our conclusion next follows in the last section of the paper.

\section{Outline of the procedure}

Let us introduce a dimensionless parameter $\epsilon$ to identify all the damping terms of Eq.(1). We are concerned in the present study by the cases where 
these terms are sufficiently small as compared to the other terms. So we assume that $0<\epsilon \ll 1$. Now, let $\tau=\tau(t)$ be the phase of oscillations for the oscillator modeled by Eq.(1), and $\rho=\rho(t)$ its instantaneous amplitude. Then there exists a function $Z$ of two variables which depends on $\epsilon$, with $Z(a, b+2 \pi ; \epsilon)=Z(a, b ; \epsilon)$, such that $x(t)=Z(\rho, \tau ; \epsilon)$. We envisage $\tau(t)$ and $\rho(t)$ as solutions of the following ODEs

$$
\begin{aligned}
& \dot{\tau}=\Omega(\rho ; \epsilon), \quad \tau(0)=0, \\
& \dot{\rho}=\epsilon \xi(\rho ; \epsilon) ; \quad \rho(0)=\rho_{0}
\end{aligned}
$$

where the actually unknown functions $\Omega$ and $\xi$ are assumed to be $O(1)$ in $\epsilon$. In terms of $Z, \tau, \rho$ and their derivatives, Eq.(1) becomes

$$
\begin{aligned}
\Omega^{2} \frac{\partial^{2} Z}{\partial \tau^{2}}+\epsilon \xi\left(2 \Omega \frac{\partial^{2} Z}{\partial \rho \partial \tau}+\Omega^{\prime} \frac{\partial Z}{\partial \tau}\right) & +\epsilon^{2} \xi\left(\xi \frac{\partial^{2} Z}{\partial \rho^{2}}+\xi^{\prime} \frac{\partial Z}{\partial \rho}\right) \\
& +f\left(Z, \Omega \frac{\partial Z}{\partial \tau}+\epsilon \xi \frac{\partial Z}{\partial \rho}\right)=0 .
\end{aligned}
$$

Note that we use the prime to denote differentiation with respect to $\rho$ when the involved functions do not depend on $\tau$. Also, the arguments of the various functions will often be omitted for notational convenience as in this equation. We now propose the following iterative scheme for determining the approximate expressions of $Z, \Omega$ and $\xi$.

Under the property defined in Eq.(2a), the Fourier expansion of $Z$ will contain only odd order harmonics [19]. We express the $n^{\text {th }}(n \geq 0)$ approx- 
imation to $Z$ as

$$
\begin{aligned}
Z_{n}(\rho, \tau ; \epsilon) & =\rho \cos (\tau)-\epsilon \frac{\xi}{\Omega} \sin (\tau)+\epsilon^{2} C_{2 n+1}^{(n)}(\rho ; \epsilon)[\cos ((2 n+1) \tau)-\cos (\tau)] \\
& +\sum_{m=1}^{n-1}\left(C_{2 m+1}^{(n-1)}(\rho ; 1)+\epsilon^{2} C_{2 m+1}^{(n)}(\rho ; \epsilon)\right)[\cos ((2 m+1) \tau)-\cos (\tau)] \\
& +\epsilon^{2} \sum_{m=1}^{n} S_{2 m+1}^{(n)}(\rho ; \epsilon)[\sin ((2 m+1) \tau)-(2 m+1) \sin (\tau)]
\end{aligned}
$$

This expression should be understood as follows. The least approximate solution consists of the first two terms of Eq.(5). Then the approximate solution considered at each iteration is obtained by adding a couple of new harmonics $\left(C_{2 n+1}^{(n)}, S_{2 n+1}^{(n)}\right)$ to the previous approximation. The coefficients of the cosine terms already present in the approximation are only improved. But for simplicity those of the sine terms, as well as $\xi$ and $\Omega$, are recomputed anew $^{1}$. It should be noted that this expression satisfies the given initial conditions.

Now we substitute this expression of $Z$ into Eq.(4) and expand the resulting equation in Taylor series up to second order in $\epsilon$. This truncated $\epsilon$-series expansion is next expanded in Fourier series. Its coefficients associated with the harmonics that are present in the expression of $Z_{n}(\rho, \tau ; \epsilon)$ are equated to zero. Considering higher order harmonics first, we obtain a system of $2 n$ linear algebraic equations in the $2 n$ unknowns $\left\{C_{2 m+1}^{(n)}(\rho), S_{2 m+1}^{(n)}(\rho),(m=1, \cdots, n)\right\}$. This system is solved and its

\footnotetext{
${ }^{1}$ This avoids very cumbersome expressions (not notably more accurate) for the sought quantities.
} 
solution is then substituted in the coefficents of the fundamental harmonics $\cos (\tau)$ and $\sin (\tau)$ of our Fourier series. Equating these last coefficients to zero leads to a set of two coupled differential equations in $\Omega$ and $\xi$ : $\chi\left(\Omega, \xi, \Omega^{\prime}, \xi^{\prime} ; \epsilon\right)=\psi\left(\Omega, \xi, \Omega^{\prime}, \xi^{\prime} ; \epsilon\right)=0$. Finally, the expressions of $\Omega(\rho ; \epsilon)$ and $\xi(\rho ; \epsilon)$ defining the slow flow Eqs.(3) are determined approximately by substituting the following truncated power series in the coupled ODEs:

$$
\begin{aligned}
\Omega^{2}(\rho ; \epsilon) & =\sum_{i=0}^{n+1} \epsilon^{2 i} \Omega_{i}(\rho), \\
\xi(\rho ; \epsilon) & =\sum_{i=0}^{n} \epsilon^{2 i} \xi_{i}(\rho) .
\end{aligned}
$$

In previous approaches to the problem considered herein, the harmonic balance was used to determine the approximate solution of the undamped equation. This approximate solution was subsequently used in a combination of MMS with Galerkin projections [20] or in the harmonic balance base averaging technique [21] to obtain the slow flow. We remark in the one hand that the solution of the conservative (or undamped) system contains only cosine terms [19]. Consequently, the solution misses the sine terms that should obviously be present due to dissipative forces. On the other hand, such approaches are useless for systems whose conservative counterparts are linear, such as the van der Pol oscillator. It is also worth to point out that in our approach the coefficients $\left\{C_{2 m+1}^{(n)}(\rho), S_{2 m+1}^{(n)}(\rho),(m=1, \cdots, n)\right\}$ depend on $\Omega$ and/or $\xi$ [see Eqs.(14) and Eqs.(18)] which in turn depend 
on the damping parameters. All the coefficients of our approximate solution, Eq.(5), thus depend on the damping parameters as one can guess for Eq.(1). So our solution procedure is, at least in principle, more powerful than the existing ones.

\section{Examples}

In this section, two examples are given to illustrate the procedure outlined above.

\subsection{A quadratic nonanalytic oscillator}

First consider the following oscillator with quadratic nonanalytic restoring force $[20,22]$

$$
\ddot{x}+\epsilon \delta_{1} \dot{x}+\sigma_{2} x|x|=0 ; \quad \sigma_{2}>0 .
$$

Note that this equation has no linear term and hence that the standard methods cannot be used in determining its analytical solution. The form of the simplest approximation to the solution is obtained from Eq.(5) for $n=0$ as

$$
Z_{0}(\rho, \tau ; \epsilon)=\rho \cos (\tau)-\epsilon \frac{\xi}{\Omega} \sin (\tau) .
$$

Since this expression contains no higher order harmonics, only the coefficients of $\cos (\tau)$ and $\sin (\tau)$ of the Fourier series of Eq.(7) are needed when 
the expression of $Z_{0}(\rho, \tau ; \epsilon)$ is substituted for $x$. We recall that these coefficients are computed after expanding the equation to second order in $\epsilon$. They can be derived for each terms of Eq.(7) from the general expressions given in Appendix A, eventually setting $C_{3}^{(1)}=S_{3}^{(1)}=0$. The following equations are easily obtained in this way from the coefficients of $\cos (\tau)$ and $\sin (\tau)$, respectively:

$$
\begin{array}{r}
2 \Omega^{2} \rho\left(8 \sigma_{2} \rho-3 \pi \Omega^{2}\right)+2 \xi\left[\left(4 \sigma_{2}+3 \pi \Omega \Omega^{\prime}\right) \xi-3 \pi \Omega^{2} \xi^{\prime}\right] \epsilon^{2}=0 \\
{\left[\left(6 \pi \Omega^{2}+6 \pi \rho \Omega \Omega^{\prime}+16 \sigma_{2} \rho\right) \xi+6 \pi \delta_{1} \rho \Omega^{2}\right] \epsilon=0 .}
\end{array}
$$

To decouple these equations, we let $\Omega^{2}=\Omega_{0}+\epsilon^{2} \Omega_{1}$ and $\xi=\xi_{0}$ according to Eqs.(6). Then the zeroth order approximations to $\Omega$ and $\xi$ are found to be

$$
\begin{aligned}
& \Omega^{(0)}(\rho ; \epsilon)=\sqrt{\frac{8 \sigma_{2} \rho}{3 \pi}} \\
& \xi^{(0)}(\rho ; \epsilon)=-\frac{2 \delta_{1} \rho}{5}
\end{aligned}
$$

These expressions are exactly those obtained in [20].

The next approximation to the solution of Eq.(7) is expressed from Eq.(5) for $n=1$ as

$$
\begin{aligned}
Z_{1}(\rho, \tau ; \epsilon) & =\rho \cos (\tau)-\epsilon \frac{\xi}{\Omega} \sin (\tau) \\
& +\epsilon^{2} C_{3}^{(1)}[\cos (3 \tau)-\cos (\tau)]+\epsilon^{2} S_{3}^{(1)}[\sin (3 \tau)-3 \sin (\tau)] .
\end{aligned}
$$

By balancing $\cos (3 \tau)$ and $\sin (3 \tau)$ in the $\epsilon^{2}$-Taylor expansion of Eq.(7) after 
substitution of the above approximation for $x$ and using Appendix A, we have

$$
\begin{array}{r}
56 \sigma_{2} \rho^{2} \Omega^{2}-196 \epsilon^{2} \sigma_{2} \xi^{2}+\left(320 \sigma_{2} \rho \Omega^{2}-945 \pi \Omega^{4}\right) C_{3}^{(1)}=0 \\
56 \epsilon \sigma_{2} \rho \xi+\epsilon^{2} \Omega\left(315 \pi \Omega^{2}+32 \sigma_{2} \rho\right) S_{3}^{(1)}=0
\end{array}
$$

while the coefficients of $\cos (\tau)$ and $\sin (\tau)$ give

$$
\begin{aligned}
10 \rho \Omega^{2}\left(8 \sigma_{2} \rho-3 \pi \Omega^{2}\right)+\left[\left(30 \pi \Omega^{4}\right.\right. & \left.-128 \sigma_{2} \rho \Omega^{2}\right) C_{3}^{(1)}-30 \pi \Omega^{2} \xi \xi^{\prime} \\
& \left.+\left(40 \sigma_{2}+30 \Omega \Omega^{\prime}\right) \xi^{2}\right] \epsilon^{2}=0 \\
{\left[\left(30 \pi \Omega^{2}+30 \pi \rho \Omega \Omega^{\prime}+80 \sigma_{2} \rho\right) \xi\right.} & \left.+30 \pi \delta_{1} \rho \Omega^{2}\right] \epsilon \\
& +6 \Omega\left(32 \sigma_{2} \rho-15 \pi \Omega^{2}\right) \epsilon^{2}=0
\end{aligned}
$$

Solving Eqs.(12) for $\left\{C_{3}^{(1)}, S_{3}^{(1)}\right\}$, we obtain

$$
\begin{aligned}
& C_{3}^{(1)}=\frac{28 \sigma_{2}\left(2 \rho^{2} \Omega^{2}-7 \epsilon^{2} \xi^{2}\right)}{5 \epsilon^{2} \Omega^{2}\left(189 \pi \Omega^{2}-64 \sigma_{2} \rho\right)}, \\
& S_{3}^{(1)}=-\frac{56 \sigma_{2} \rho \xi}{\epsilon \Omega\left(32 \sigma_{2} \rho+315 \pi \Omega^{2}\right)} .
\end{aligned}
$$

The new approximate expressions of $\Omega$ and $\xi$ are obtained by substituting Eqs.(14) into Eqs.(13) and letting $\Omega^{2}=\Omega_{0}+\epsilon^{2} \Omega_{1}+\epsilon^{4} \Omega_{2}$ and $\xi=\xi_{0}+\epsilon^{2} \xi_{1}$ to proceed to decoupling. It should be pointed out here that the equation that determines the zeroth order approximation to $\Omega$ always has a unique solution, $\Omega^{(0)}$. In contrast the corresponding equation for higher order iterations may have more than one solution. In this case, the solution 
retained is the one for which

$$
\lim _{\rho \rightarrow 0} \frac{\Omega^{(n)}(\rho ; 0)}{\Omega^{(n-1)}(\rho ; 0)} \approx 1 .
$$

Using floating points numbers to simplify the notation, they read

$$
\begin{aligned}
\Omega^{(1)}(\rho ; \epsilon) & =\left[0.83526936259440433 \sigma_{2} \rho+0.010426866870164911 \delta_{1}^{2} \epsilon^{2}\right. \\
& \left.-0.0012192060202053301 \frac{\delta_{1}^{4}}{\sigma_{2} \rho} \epsilon^{4}\right]^{\frac{1}{2}} \\
\xi^{(1)}(\rho ; \epsilon) & =-0.39172301863602720 \delta_{1} \rho \\
& -0.0026671295819383208 \frac{\delta_{1}^{3}}{\sigma_{2}} \epsilon^{2}
\end{aligned}
$$

We limit ourselves at this level. Then, setting $\epsilon=1$ in the various steps, the final approximate solution of Eq.(7) is given by Eqs.(11)-(14) in which $\Omega \equiv \Omega^{(1)}(\rho(t)), \xi \equiv \xi^{(1)}(\rho(t)) ; \rho(t)$ and $\tau(t)$ being solutions of Eqs.(3) with $\Omega(\rho ; 1)$ and $\xi(\rho ; 1)$ taken from Eqs.(15). This approximate semianalytical ${ }^{2}$ solution is compared to the result of direct numerical integration in Fig. 1. Very good matches are observed for small to order $O(1)$ values of the damping coefficient.

\subsection{The Duffing oscillator}

Our second example is a damped Duffing oscillator

$$
\ddot{x}+\epsilon \delta_{1} \dot{x}+\sigma_{1} x+\sigma_{3} x^{3}=0 .
$$

\footnotetext{
${ }^{2}$ Eqs.(3) are integrated numerically
} 
In view of condition $(2 \mathrm{~b})$, we assume that either $\sigma_{1}$ is nonnegative or that $\sigma_{3}>0$ if $\sigma_{1}=0$. Note again that the standard methods do not apply in the latter case.

Using again the Fourier series of the various terms of Eq.(16) provided in Appendix A and proceeding as in the preceding subsection, it is easy to verify that the zeroth order approximation to $\Omega$ and $\xi$ obtained for the Duffing oscillator starting from Eq.(8) are given by

$$
\begin{aligned}
& \Omega^{(0)}(\rho ; \epsilon)=\sqrt{\sigma_{1}+\frac{3 \sigma_{3} \rho^{2}}{4}-\epsilon^{2} \frac{\delta_{1}^{2}\left(4 \sigma_{1}+3 \sigma_{3} \rho^{2}\right)^{2}\left(8 \sigma_{1}-9 \sigma_{3} \rho^{2}\right)}{\left(8 \sigma_{1}+9 \sigma_{3} \rho^{2}\right)^{3}}}, \\
& \xi^{(0)}(\rho ; \epsilon)=-\frac{\delta_{1} \rho\left(4 \sigma_{1}+3 \sigma_{3} \rho^{2}\right)}{8 \sigma_{1}+9 \sigma_{3} \rho^{2}} .
\end{aligned}
$$

It is interesting to note that in contrast to the first example, the expression of $\Omega$ already depends on the damping parameter at this level. Furthermore, in the limit $\sigma_{3}=0$, we recover the exact result for the damped linear oscillator as expected.

Looking now for an approximate solution of the form of Eq.(11), one easily finds that

$$
\begin{aligned}
C_{3}^{(1)} & =\frac{\sigma_{3} \rho\left(\Omega^{2} \rho^{2}-3 \epsilon^{2} \xi^{2}\right)}{\epsilon^{2} \Omega^{2}\left(36 \Omega^{2}-4 \sigma_{1}-3 \sigma_{3} \rho^{2}\right)}, \\
S_{3}^{(1)} & =-\frac{3 \sigma_{3} \rho^{2} \xi}{\epsilon \Omega\left(36 \Omega^{2}-4 \sigma_{1}+3 \sigma_{3} \rho^{2}\right)} .
\end{aligned}
$$

For the full Duffing oscillator (16), the refinement process for $\Omega$ and $\xi$ at this level leads to too cumbersome expressions. For simplicity, we give these 
expressions below in the interesting case where $\sigma_{1}=0$ :

$$
\begin{aligned}
\Omega^{(1)}(\rho ; \epsilon) & =\left[0.71553172956504432 \sigma_{3} \rho^{2}+0.13397016524712223 \delta_{1}^{2} \epsilon^{2}\right. \\
& \left.-0.00074558383429463651 \frac{\delta_{1}^{4}}{\sigma_{3} \rho^{2}} \epsilon^{4}\right]^{\frac{1}{2}} \\
\xi^{(1)}(\rho ; \epsilon) & =-0.31822443946079998 \delta_{1} \rho \\
& -0.036288655675012770 \frac{\delta_{1}^{3}}{\sigma_{3} \rho} \epsilon^{2}
\end{aligned}
$$

In Figs. 2-3, the approximate semianalytical solution Eqs.(11)-(18) is compared to the exact numerical integration of Eq.(16). Note here that $\Omega$ and $\xi$ are approximated in Eq.(3) and Eqs.(18) by the expressions in Eqs.19 when $\sigma_{1}=0$ or by those in Eqs.(17) if $\sigma_{1} \neq 0$. Again excellent matches are observed.

\section{Conclusion and remarks}

This paper has dealt with the derivation of the slow flow equations for some class of autonomous damped single degree of freedom nonlinear oscillator. We have specifically been interested by systems undergoing symmetric restoring forces and damping. Our approach is based on the linearized harmonic balance due to $\mathrm{Wu}$ and and co-workers. Their technique is used to compute the coefficients of the harmonics of an assumed form of the solution and to derive a system of two coupled ODEs related to the slow 
flow. A power series procedure is next used to decouple the coupled system and to obtain the slow flow.

As any approximate method, this procedure has its own limitations. Namely, although the iterative process can in principe be repeated any number of time, one will in practice be limited by the size of the system of linear algebraic equations to be solved; which is two times greater than that of the undamped system. Hence, the refinement might perhaps be repeated only once. Nevertheless, our examples show not only that the procedure complements the standard ones in particular for essentially nonlinear problems, but also that the accuracy obtained with even a single refinement is very excellent for reasonably small damping.

An interesting point under investigation is the generalization of the linearized harmonic balance to the analysis of coupled oscillators and, specifically to the determination of high-amplitude waves' envelope equations in one dimensional lattice. 


\section{A Appendix}

In this appendix, we provide the Fourier series of the $\epsilon^{2}$-series expansion of the various terms of our examples for $x$ approximated by Eq.(11). The Fourier series for $x$ approximated by Eq.(8) is obviously obtained by setting $C_{3}^{(1)}=S_{3}^{(1)}=0$.

$$
\begin{gathered}
Z_{1}(\rho, \tau ; \epsilon)=\left(\rho-\epsilon^{2} C_{3}^{(1)}\right) \cos (\tau)-\left(\epsilon \frac{\xi}{\Omega}-3 \epsilon^{2} S_{3}^{(1)}\right) \sin (\tau) \\
+\epsilon^{2} C_{3}^{(1)} \cos (3 \tau)+\epsilon^{2} S_{3}^{(1)} \sin (3 \tau) \\
\dot{Z}_{1}(\rho, \tau ; \epsilon)=-3 \epsilon^{2} \Omega S_{3}^{(1)} \cos (\tau)-\left[\rho \Omega+\epsilon^{2}\left(\frac{\xi \xi^{\prime}}{\Omega}-\frac{\xi^{2} \Omega^{\prime}}{\Omega^{2}}-C_{3}^{(1)} \Omega\right)\right] \sin (\tau) \\
+3 \epsilon^{2} \Omega S_{3}^{(1)} \cos (3 \tau)-3 \epsilon^{2} \Omega C_{3}^{(1)} \sin (3 \tau)+O\left(\epsilon^{3}\right) \\
\quad-\epsilon\left[\xi\left(\Omega+\rho \Omega^{\prime}\right)-3 \epsilon^{2} \Omega^{2} S_{3}^{(1)}\right] \sin (\tau) \\
\ddot{Z}_{1}(\rho, \tau ; \epsilon)=-\left[\rho \Omega^{2}+\epsilon^{2}\left(\xi \xi^{\prime}-\frac{\xi^{2} \Omega^{\prime}}{\Omega}-\Omega^{2} C_{3}^{(1)}\right)\right] \cos (\tau) \\
\quad-9 \epsilon^{2} \Omega^{2} C_{3}^{(1)} \cos (3 \tau)-9 \epsilon^{2} \Omega^{2} S_{3}^{(1)} \sin (3 \tau)+O\left(\epsilon^{3}\right) \\
Z_{1}^{3}=\left[\frac{3 \rho^{3}}{4}+\epsilon^{2}\left(\frac{3 \rho \xi^{2}}{4 \Omega^{2}}-\frac{3 \rho^{2}}{2} C_{3}^{(1)}\right)\right] \cos (\tau)-\left[\epsilon \frac{3 \rho^{2} \xi}{4 \Omega}+\epsilon^{2} \frac{3 \rho^{2}}{2} S_{3}^{(1)}\right] \sin (\tau) \\
+\left[\frac{\rho^{3}}{4}+\epsilon^{2}\left(\frac{3 \rho^{2}}{4} C_{3}^{(1)}-\frac{3 \rho \xi^{2}}{4 \Omega^{2}}\right)\right] \cos (3 \tau) \\
-\left[\epsilon \frac{3 \rho^{2} \xi}{4 \Omega}+\epsilon^{2} \frac{3 \rho^{2}}{4} S_{3}^{(1)}\right] \sin (3 \tau) \\
+\frac{3}{4} \epsilon^{2} \rho^{2} C_{3}^{(1)} \cos (5 \tau)+\frac{3}{4} \epsilon^{2} \rho^{2} S_{3}^{(1)} \sin (5 \tau)+O\left(\epsilon^{3}\right)
\end{gathered}
$$


To give the Taylor series of $Z_{1}\left|Z_{1}\right|$, we note that if

$$
g(x)=x|x|
$$

then the first and second derivatives of $g$ read

$$
\begin{aligned}
g^{\prime}(x) & =2|x| \\
g^{\prime \prime}(x) & =2 \operatorname{sign}(x)=\frac{|x|}{x}, \quad x \neq 0
\end{aligned}
$$

Thus

$$
\begin{aligned}
Z_{1}\left|Z_{1}\right|= & \rho \cos (\tau)|\rho \cos (\tau)|-2 \epsilon \frac{\xi}{\Omega} \sin (\tau)|\rho \cos (\tau)|+\epsilon^{2} \frac{\xi^{2} \sin ^{2}(\tau)}{\Omega^{2} \rho \cos (\tau)}|\rho \cos (\tau)| \\
+ & 2 \epsilon^{2}\left[C_{3}^{(1)}(\cos (3 \tau)-\cos (\tau))+S_{3}^{(1)}(\sin (3 \tau)-3 \sin (\tau))\right]|\rho \cos (\tau)| \\
+ & O\left(\epsilon^{3}\right) \\
Z_{1}\left|Z_{1}\right| & =\frac{1}{\pi}\left[\frac{8 \rho^{2}}{3}+\epsilon^{2}\left(\frac{4 \xi^{2}}{3 \Omega^{2}}-\frac{64 \rho}{15} C_{3}^{(1)}\right)\right] \cos (\tau) \\
& -\left(\epsilon \frac{8 \rho \xi}{3 \Omega}+\epsilon^{2} \frac{32 \rho}{5} S_{3}^{(1)}\right) \sin (\tau) \\
+ & \frac{1}{\pi}\left[\frac{8 \rho^{2}}{15}+\epsilon^{2}\left(\frac{64 \rho}{21} C_{3}^{(1)}-\frac{28 \xi^{2}}{15 \Omega^{2}}\right)\right] \cos (3 \tau) \\
- & \frac{1}{\pi}\left(\epsilon \frac{8 \rho \xi}{5 \Omega}+\epsilon^{2} \frac{32 \rho}{35} S_{3}^{(1)}\right) \sin (3 \tau)+O\left(\epsilon^{3}\right)
\end{aligned}
$$




\section{References}

[1] V. P. Lukomsky, V. B. Bobkov, Asymptotic expansions of the periodic solutions of nonlinear evolution equations, Nonlinear Dynamics 16 (1998) 1-21.

[2] B. S. Wu, P. S. Li, A method for obtaining approximate analytic periods for a class of nonlinear oscillators, Meccanica 36 (2001) 167176.

[3] C. W. Lim, B. S. Wu, A new analytical approach to the Duffingharmonic oscillator, Physics Letters A 311 (2003) 365-373.

[4] J.-H. He, Modified Lindstedt-Poincare methods for some strongly non-linear oscillations, Part I: expansion of a constant, International Journal of Non-Linear Mechanics 37 (2002) 309-314.

[5] P. Amore, A. Aranda, Presenting a new method for the solution of nonlinear problems, Physics Letters A 316 (2003) 218-225.

[6] B. S. Wu, C. W. Lim, Large amplitude non-linear oscillations of a general conservative system, International Journal of Non-Linear Mechanics 39 (2004) 859-870. 
[7] P. Amore, A. Aranda, Improved Lindstedt-Poincaré method for the solution of nonlinear problems, Journal of Sound and Vibration 283 (2005) 1115-1136.

[8] B. S. Wu, C. W. Lim, W. P. Sun, Improved harmonic balance approach to periodic solutions of non-linear jerk equations, Physics Letters A 354 (2006) 95-100.

[9] B. S. Wu, W. P. Sun, C. W. Lim, An analytical approximate technique for a class of strongly non-linear oscillators, International Journal of Non-Linear Mechanics 41 (2006) 766-774.

[10] C. W. Lim, B. S. Wu, W. P. Sun, Higher accuracy analytical approximations to the Duffing-Harmonic oscillator, Journal of Sound and Vibration 296 (2006) 1039-1045.

[11] V. Marinca, N. Herisanu, A modified iteration perturbation method for some nonlinear oscillation problems, Acta Mechanica 184 (2006) 231-242.

[12] H. Hu, Solution of a quadratic nonlinear oscillator by the method of harmonic balance, Journal of Sound and Vibration 293 (2006) 462-468. 
[13] H. Hu, Solutions of nonlinear oscillators with fractional powers by an iteration procedure, Journal of Sound and Vibration 294 (2006) $608-614$.

[14] H. Hu, Solutions of a quadratic nonlinear oscillator: Iteration procedure, Journal of Sound and Vibration 298 (2006) 462-468.

[15] R. E. Mickens, Iteration method solutions for conservative and limitcycle $x^{1 / 3}$ force oscillators, Journal of Sound and Vibration 292 (2006) 964-968.

[16] A. H. Nayfeh, D. T. Mook, Nonlinear Oscillations, Wiley, New York, 1979.

[17] S. B. Yamgoué, T. C. Kofané, The subharmonic Melnikov theory for damped and driven oscillators revisited, International Journal of Bifurcation and Chaos 12, (2002) 1915-1923.

[18] R. E. Mickens, Comments on the method of harmonic balance, Journal of Sound and Vibration 94 (1984) 456-460.

[19] R. E. Mickens, Fourier representations for periodic solutions of oddparity systems, Journal of Sound and Vibration 258 (2002) 398401. 
[20] S. L. Das, A. Chatterjee, Multiple scales via Galerkin projections: approximate asymptotics for strongly nonlinear oscillations, Nonlinear Dynamics 32 (2003) 161-186.

[21] A. Chatterjee, Harmonic balance based averaging: approximate realizations of an asymptotic technique, Nonlinear Dynamics 32 (2003) 323-343.

[22] K. Nandakumar, A. Chatterjee, Higher-order pseudoaveraging via harmonic balance for strongly nonlinear oscillations, Journal of $V i$ bration and Acoustics 127 (2005) 416-419. 


\section{FIGURE CAPTIONS}

Figure 1. Comparison of the approximate analytical (dashed line) waveforms to the corresponding exact numerical (solid line) ones for the quadratic nonlinear oscillator (7) for $\sigma_{2}=1$. Initial conditions are $x(0)=\rho(0)=3, \dot{x}(0)=\tau(0)=0 . \quad$ (a) $\delta_{1}=0.1$; (b) $\delta_{1}=0.5 ;$ (c) $\delta_{1}=0.75 ;(\mathrm{d}) \delta_{1}=1$.

Figure 2. Comparison of the approximate analytical (dashed line) waveforms to the corresponding exact numerical (solid line) ones for the Duffing oscillator (16) for $\sigma_{1}=\sigma_{3}=1$. Initial conditions are $x(0)=$ $\rho(0)=3, \dot{x}(0)=\tau(0)=0$. (a) $\delta_{1}=0.1$; (b) $\delta_{1}=0.5$; (c) $\delta_{1}=0.75$; (d) $\delta_{1}=1$.

Figure 3. Same as in Fig 2, with $\sigma_{1}=0$. 

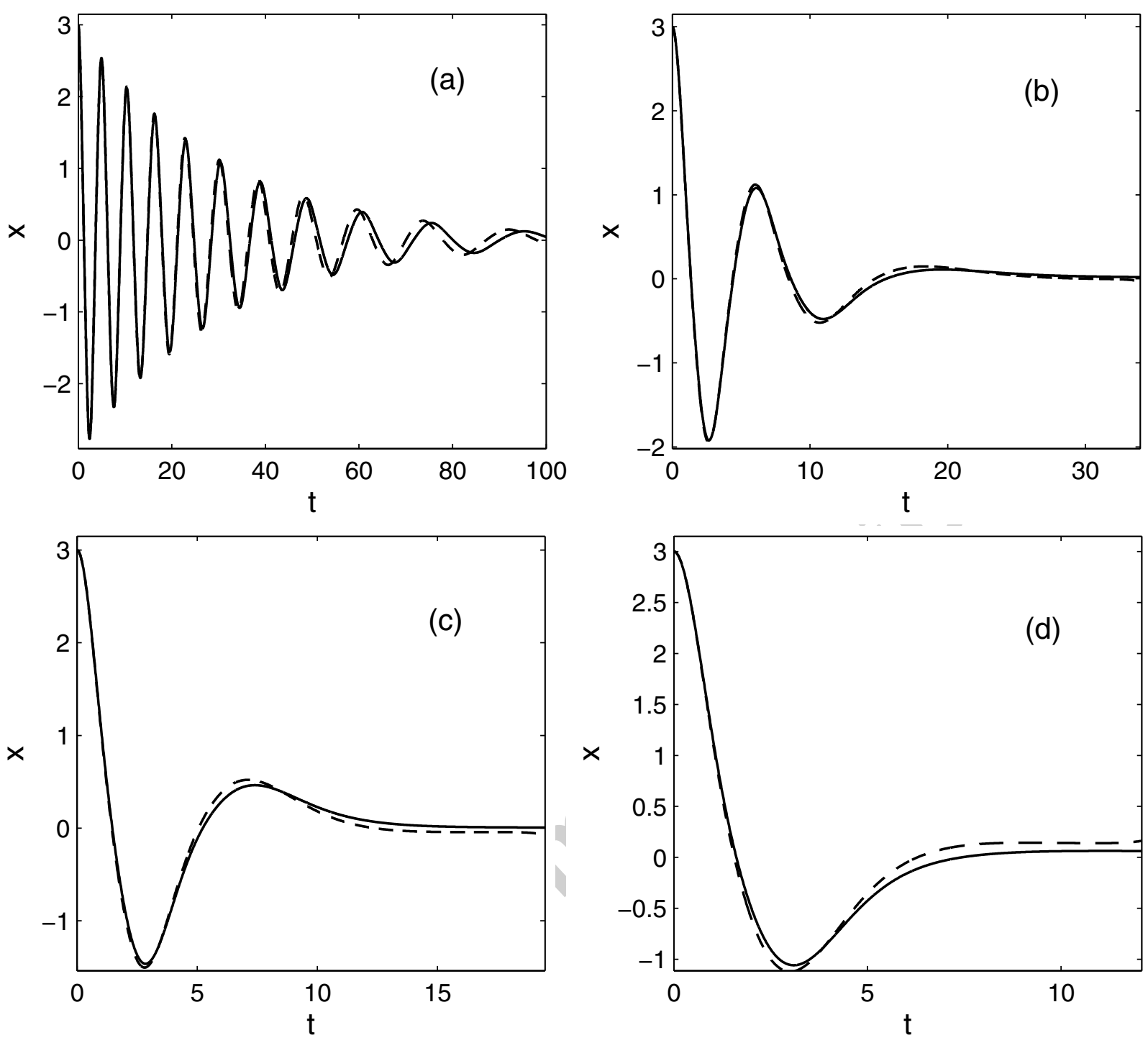

Figure 1: S. B. Yamgoué and T. C. Kofané 

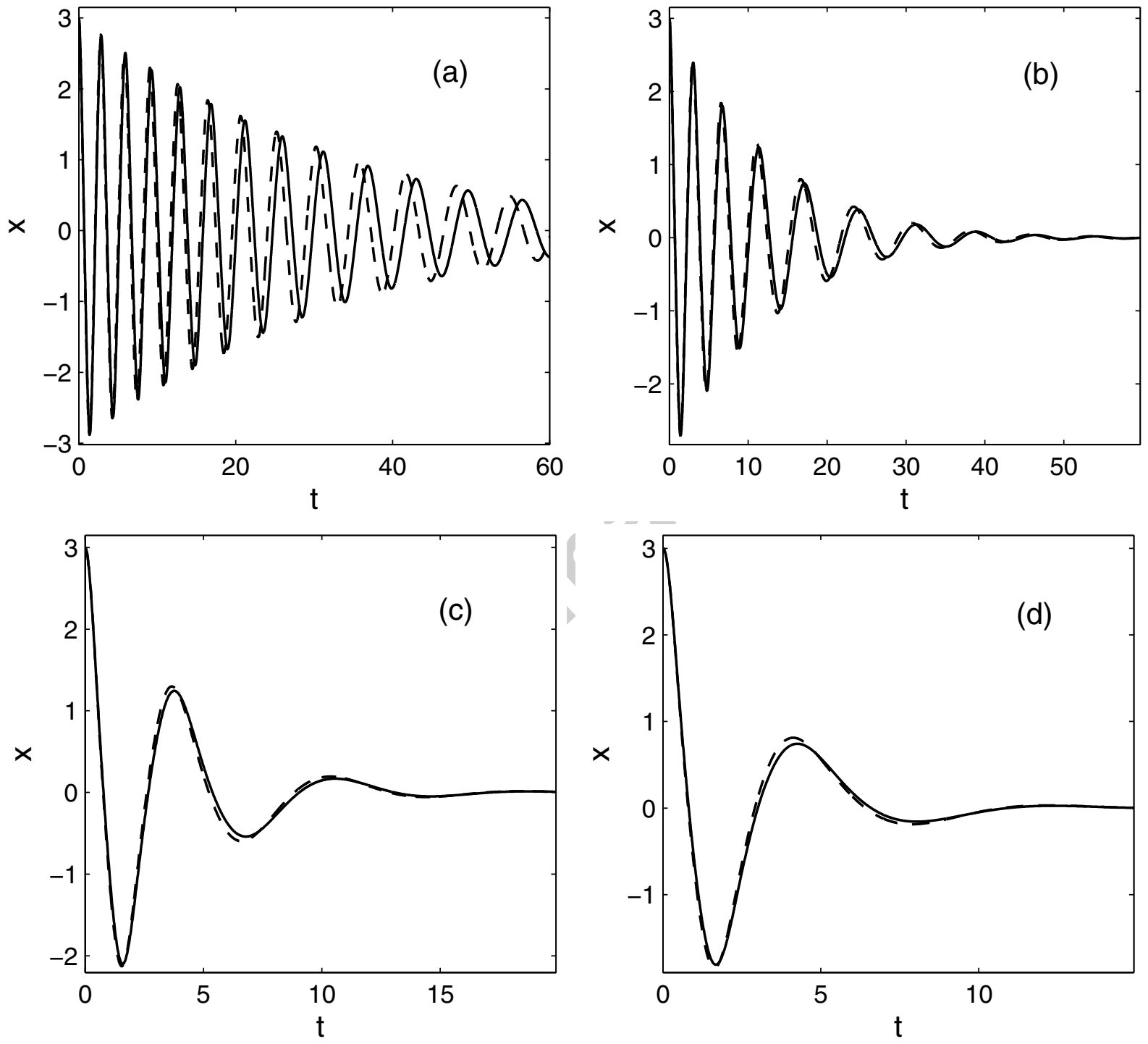

Figure 2: S. B. Yamgoué and T. C. Kofané 

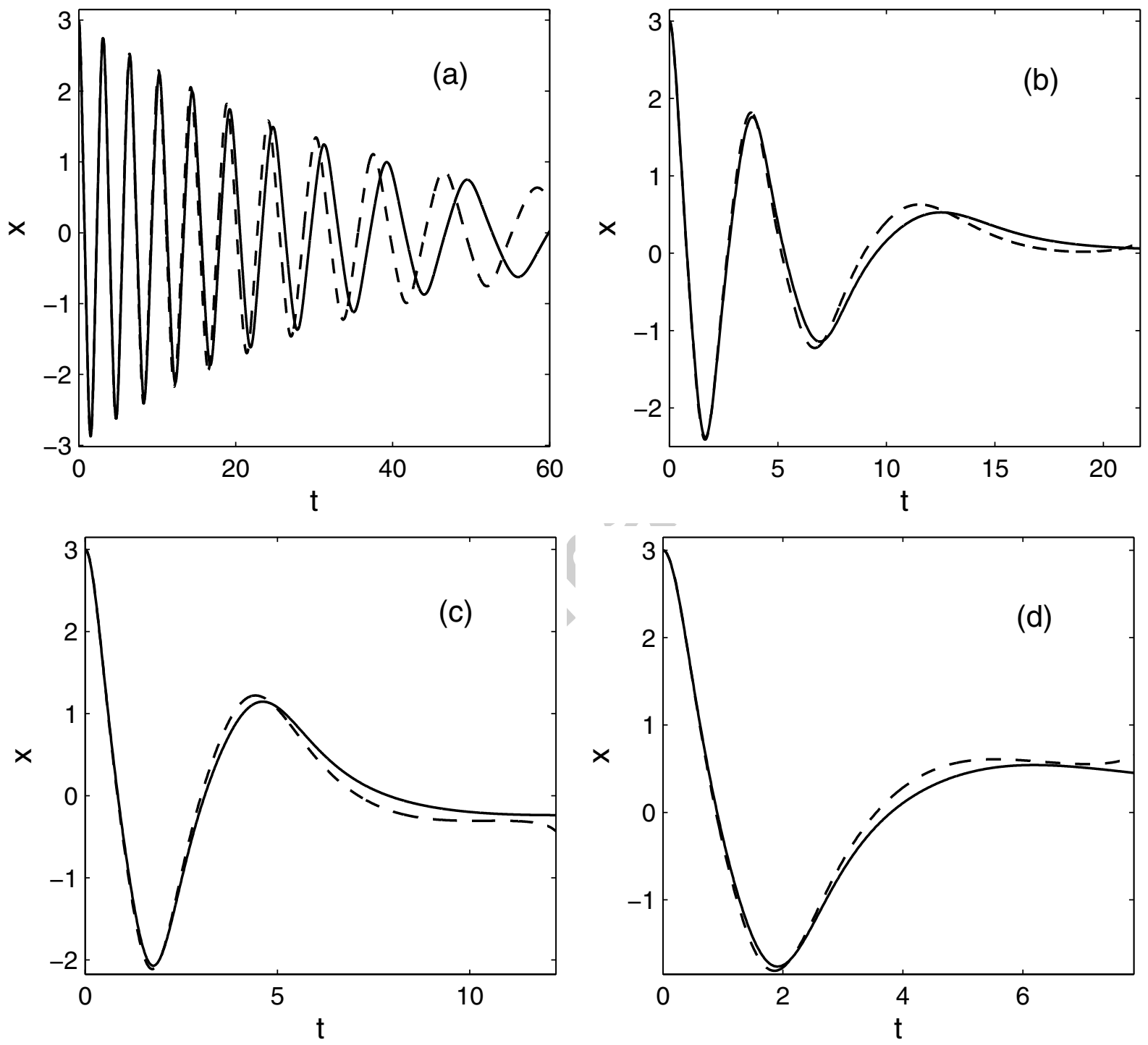

Figure 3: S. B. Yamgoué and T. C. Kofané 